\title{
Effectiveness of Ultrasonography in Detection of Loco- regional Recurrence of Breast Cancer
}

\author{
Mai Sayed Khalifa ${ }^{1}$, MohmmedSoliman Gaber ${ }^{1}$, Walid Omar Soliman ${ }^{2}$ \\ ${ }^{1}$ Department ofClinical Oncology \& Nuclear Medicine,Sohag Faculty of Medicine, \\ Sohag University. \\ ${ }^{2}$ Department of Nuclear Medicine, National cancer institute-Cairo.
}

\section{Introduction}

Breast cancer is the most common type of cancer and the second leading cause of cancer related death among women ${ }^{(\mathbf{1})}$. The estimated incidence in 2018 was 2.088 million of new cases in the world ${ }^{(2)}$. Breast cancer was the most common cancer among women and the third leading cause of cancer related death in Egypt ${ }^{(3)}$.

Loco-regional recurrence of breast cancer (LRR) is defined asrecurrence in the ipsilateral breast or axillary lymph nodes $^{(4)}$.It is a major clinical manifestation andmay occur in 5-27\% of patients (5). Early detection and treatment of isolated LRR before symptomatic onset may have a beneficial effect on the prognosis, by improving local treatment feasibility rate and by avoiding the situation of uncontrollable loco-regional disease ${ }^{(6)}$.

Breast US has been popularly used to characterize lesions and differentially diagnose breast masses as an adjunctive tool to mammography, particularly in women with dense breasts ${ }^{(7)}$.It is a widely available, relatively inexpensive imaging method that is easy to perform, has no radiation hazards, does not require a contrast agent, and enables biopsy under image guidance ${ }^{(\mathbf{8})}$.

Several studies have shown surveillance results of US applied to women who were treated for breast cancer. The reported cancer detection rates were $1.7-5.1 \%$ per patient, and the positive predictive value was 21.5$52.6 \%$, with percentages varying according to the area involved ${ }^{(9)}$.Although US may have its strong points for visualizing areas that cannot be approached by mammography or provide additional information regarding differentiation between postoperative changes and LRR, little evidence suggests whether US is effective and beneficial for improving survival of patients with breast cancer and the role of US in post-treatment surveillance programs has yet to be investigated ${ }^{(\mathbf{9})}$.

This study was designed to evaluate the effectiveness of US imaging in diagnosis of LRR of breast cancer.

\section{Patients and Methods:}

The Ethical and Research committees at Faculty of Medicine, Sohag University approved this prospective study. All participants assigned an informed written consent.The study included 45 female patients with histopathologically proven breast cancer managed by mastectomy +/- chemotherapy and/or radiotherapy with post treatment tumor free interval.

\section{Exclusion criteria:}

Patients with proved other malignancy (Double primary) were excluded. Pregnant females as well aspatients with proved LRR and/or distant metastasis were also excluded.

\section{Study design:}

I- History:Detailed history was obtained from all patients with the help of their data sheets. The pathological reports as regards the initial type of cancer and its grading, tissue infiltration, and axillary lymph nodes involvement, as well as the expressed receptors (ER, PR, HER2, and Ki67) 
SOHAG MEDICAL JOURNAL

Vol. 23 No.2 Apr 2019
Effectiveness of Ultrasonography in Detection

Mai Sayed Khalifa.et al

were recorded. The post-operative therapeutic history (Chemotherapy and/or radiotherapy) wasdocumented.

II- Sono-mammography: Breast US was done for all patients by an expert radiologist with a high frequency linear array transducer (L8 $14 \mathrm{MHz}$ ). The breast was examined for any nodules or cysts. The shape of the solid lesions, boundary, echogenicity, calcification, enhancing or decreasing of post-lesion and color Doppler signal in breast were observed. Any thickening or nodules in the chest wall was recorded. The axilla was examined for any abnormality in the axillary lymph nodes. The location, size, shape, internal echo, lymph hilum, cortico-medullary structure, and flow pattern of lymph node were explored. Lymph nodes were categorized as suspicious if they exhibited one or more of the following characteristics: overall enlargement, cortical thickening, or

\section{Results}

The mean age \pm SD of the patients was $58.47 \pm 9.1$ years (Range $=40-77$ years). The vast majority of the patients had either CBS or MRM (18/45; $40 \%$ for each). Radical mastectomy was done in 8 cases (17.8\%). Around half of the cases (22 cases, $49 \%$ ) had isolated left sided tumor, with 16 cases $(35.5 \%)$ had isolated left sided tumor.

\section{Sono-mammography findings:}

Axillary lymph nodes were detected abnormal in 10 cases $(22.2 \%)$. Breast cyst in one case and breast nodules were found in 12 cases (26.7\%), and chest wall nodule or thickening was detected in 8 cases $(17.8 \%)$.

\section{Sonomammography performance in detecting LRR:}

By comparing the sonomammography for signs of recurrence with the results of biopsy or follow up; it was found that US accuracy was71.46\%, sensitivity (69.23\%), and specificity $(73.68 \%)$ (Table 1

\begin{tabular}{|c|c|l|}
\hline & Sonography & Biopsy or follow up \\
\hline True positive (TP), $\mathbf{n}$ & 18 & \\
\hline True negative (TN), $\mathbf{n}$ & 14 & \\
\hline False positive (FP), $\mathbf{n}$ & 5 & \\
\hline False negative (FN), $\mathbf{n}$ & 8 & \\
\hline Sensitivity (\%) & 69.23 & \\
\hline Specificity (\%) & 73.68 & \\
\hline Positive predictive value (\%) & 78.26 & \\
\hline Negative predictive value (\%) & 63.64 & \\
\hline Accuracy (\%) & 71.46 & \\
\hline
\end{tabular}

Table 1:Performance of ultrasonography in detecting LRR. 


\section{Discussion}

LRR of breast canceris a major clinical manifestation andmay occur in $5-27 \%$ of patients ${ }^{(5)}$. Early detection of recurrence has a value in decreasing the breast cancer associated morbidity and mortality (10). Breast USmay have a role in diagnosis of LRR of breast cancer with variable results in the previous studies $^{(9)}$.

In the present study; sensitivity of US in detection of LRR of breast cancer was $69.23 \%$. This was similar to previous reports byShin et al. ${ }^{(11)}$ $(70.59 \%)$ and Lamuraglia et $\boldsymbol{a l}^{(\mathbf{1 2})}$ (66.67\%).However; this sensitivity was less than previously reported by Winehouse et al. ${ }^{(\mathbf{1 3})}(93.75 \%)$,Ternier et al. ${ }^{(14)}$ (86.54\%),Yilmaz et al. ${ }^{(15)}$ (90\%), Stuhrmann et al. ${ }^{(16)}$ (94\%), andRiebe et al. $^{(\mathbf{1 7})}(90.91 \%)$. This may be related to the use of Contrastenhanced colour Doppler with more sensitivity

In the current study; specificity of US in detection of LRR of breast cancer was $73.68 \%$. This was similar to previous reports byWinehouse et al. $^{\left({ }^{(13)}\right.}(66.67 \%)$, Ternier et al. ${ }^{(14)}$ $(72.55 \%)$, Stuhrmann et al. ${ }^{(16)}$ (71.69\%), andRiebe et al. ${ }^{(17)}$ (68.75 $\%)$. However; this specificity was less

than previously reported byYilmaz et al. $^{(\mathbf{1 5 )}}(88.24 \%)$, Shin et al. ${ }^{(11)}(98.29$

$\%)$. This may be related to the combined use of clinical examination and mammography results. Higher specificity was also reported byLamuraglia et $\boldsymbol{a l}^{(\mathbf{1 2})}(97.5 \%)$ and this may be related to the use of Doppler US with perfusion software and contrast agent injection

In this study; accuracy of US in detection of LRR of breast cancer was $71.46 \%$. This was similar to previous $\begin{array}{lrl}\text { reports by } & \text { Winehouse } & \text { et } \\ \text { al. }^{(13)}(74.14 \%), & \text { Ternier et al. }\end{array}$
(79.61\%), Stuhrmann et al. ${ }^{(16)}(75 \%)$, Riebe et al. ${ }^{(17)}(77.78 \%)$.

Higher accuracy was previously reported by Yilmaz et al. ${ }^{\text {(15) }}$ (88.89 $\%)$, Shin et al. ${ }^{(11)}$ (97.82\%). This difference may be explained by the combined use of clinical examination and mammography results.Moreover;Lamuraglia et al. ${ }^{(12)}$ also reported higher accuracy (90\%). They used Doppler US with perfusion software and contrast agent injection for more accurate detection of LRR (12)

The results of this study confirms the added value of the use of breast US for detection of recurrence of breast cancer. However; the use of more than one modality of diagnosis may give more accurate result

\section{Referrences}

1.Taghipour M, Wray R, Sheikhbahaei S, Wright JL and Subramaniam RM: FDG Avidity and Tumor Burden: Survival Outcomes for Patients With Recurrent Breast Cancer.AJR Am J Roentgenol. 2016; 206(4): 846-55.

2. Bray F, Ferlay J, Soerjomataram I, Siegel RL, Torre LA: Global cancer statistics 2018: GLOBOCAN estimates of incidence and mortality worldwide for 36 cancers in 185 countries. CA Cancer J Clin. 2018.

3. Elatar I, Cancer registration, NCI Egypt 2001. Cairo, Egypt, National Cancer Institute 2002. 2002.

4. Ahmad A: Pathways to breast cancer recurrence.ISRN Oncol. 2013; 2013: 290568.

5. Jung KW, Won YJ, Kong HJ and Lee ES: Cancer Statistics in Korea: Incidence, Mortality, Survival, and Prevalence in 2015.Cancer Res Treat. 2018; 50(2): 303-316.

6. Kim SJ, Moon WK, Cho $\mathbf{N}$ and Chang JM: The detection of recurrent breast cancer in patients with a history of breast cancer surgery: comparison of clinical breast examination, mammography and 
ultrasonography.Acta Radiol. 2011; 52(1): 15-20.

7. Kim MJ, Kim EK, Kwak JY, Park BW, Kim SI: Role of sonography in the detection of contralateral metachronous breast cancer in an Asian population.AJR Am $J$ Roentgenol. 2008; 190(2): 476-80.

8. Kim MJ, Kim EK, Kwak JY, Park BW, Kim SI: Sonographic surveillance for the detection of contralateral metachronous breast cancer in an Asian population. AJR Am J Roentgenol. 2009; 192(1): 221-8.

9. Suh YJ, Kim MJ, Kim EK, Moon HJ, Kim SI: Value of ultrasound for postoperative surveillance of asian patients with history of breast cancer surgery: a single-center study.Ann Surg Oncol. 2013; 20(11): 3461-8.

10. Champion L, Brain E, Giraudet AL, Le Stanc E, Wartski M: Breast cancer recurrence diagnosis suspected on tumor marker rising: value of whole-body 18FDG-PET/CT imaging and impact on patient management.Cancer. 2011; 117(8): 1621-9.

11. Shin JH, Han BK, Choe YH, Nam SJ, Park W: Ultrasonographic detection of occult cancer in patients after surgical therapy for breast cancer.J Ultrasound Med. 2005; 24(5): 643-9.
12. Lamuraglia M, Lassau N, Garbay JR, Mathieu MC, Rouzier R: Doppler US with perfusion software and contrast medium injection in the early evaluation of radiofrequency in breast cancer recurrences: a prospective phase II study.Eur $J$ Radiol. 2005; 56(3): 376-81.

13. Winehouse J, Douek M, Holz K, Madjar H, Gillams A: Contrastenhanced colour Doppler ultrasonography in suspected breast cancer recurrence. Br J Surg. 1999; 86(9): 1198-201.

14. Ternier F, Houvenaeghel G, Lecrivain F, Brigand BL, Margain D: Computed tomography in suspected local breast cancer recurrence.Breast Cancer Res Treat. 2006; 100(3): 247-54.

15. Yilmaz MH, Esen G, Ayarcan Y, Aydogan F, Ozguroglu M: The role of US and MR imaging in detecting local chest wall tumor recurrence after mastectomy.Diagn Interv Radiol. 2007; 13(1): 13-8.

16. Stuhrmann $M$, Aronius $R$ and Schietzel M: Tumor vascularity of breast lesions: potentials and limits of contrast-enhanced Doppler sonography.AJR Am J Roentgenol. 2000; 175(6): 1585-9.

17. Riebe E, Gunther K, Schulz K, Kohler G, Schimming A: Recurrent disease after breast preserving therapy (BPT) and radiation therapy for breast cancer--diagnostic yield of palpation, mammography and ultrasonography.Ultraschall Med. 2007; 28(4): 394-400. 